\title{
Timing of Arthrocentesis in the Management of Temporomandibular Disorders: A Systematic Review and Meta-analysis
}

Dion Tik Shun LI ${ }^{1}$, Natalie Sui Miu WONG ${ }^{1}$, Samantha Ka Yan $\mathrm{LI}^{2}$, Colman Patrick MCGRATH $^{3}$, Yiu Yan LEUNG ${ }^{1}$

${ }^{1}$ Oral and Maxillofacial Surgery, Faculty of Dentistry, The University of Hong Kong, Hong Kong

${ }^{2}$ Faculty of Dentistry, The University of Hong Kong, Hong Kong

${ }^{3}$ Applied Oral Sciences \& Community Dental Care, Faculty of Dentistry, The

University of Hong Kong, Hong Kong

\section{Corresponding author}

Dr. Yiu Yan Leung

Oral and Maxillofacial Surgery

34 Hospital Road, Hong Kong

Tel: +85228590511

Fax: +85228575570

E-mail: mikeyyleung@hku.hk

Funding: None

Key words: temporomandibular joint disorders, arthrocentesis, TMJ, arthralgia, lavage 


\begin{abstract}
The aim of this study was to assess the best timing to perform arthrocentesis in the management of temporomandibular disorders with regards to conservative treatment. A systematic search based on PRISMA guidelines, including a computer search with specific keywords, reference list search, and manual search was done. Relevant articles were selected after 3 search rounds for final review based on 6 predefined inclusion criteria, followed by a round of critical appraisal. Eleven publications, including 5 randomized controlled trials and 6 prospective clinical studies informed this review. The studies were divided into 3 groups based on the timing of arthrocentesis: 1). Arthrocentesis as the initial treatment, 2). Early arthrocentesis, and 3). Late arthrocentesis. Meta-analyses compared the efficacy of improvement in mouth opening and pain reduction in the 3 groups. The results were statistically significant and favoured early arthrocentesis, followed by late arthrocentesis in terms of both improvements in mouth opening and pain reduction. All 3 groups showed improvement in mouth opening and pain reduction. We conclude that early arthrocentesis may be able to produce the best clinical results, while arthrocentesis before attempting conservative treatment may produce less favourable outcomes.
\end{abstract}




\section{INTRODUCTION}

Temporomandibular disorders (TMDs) are a group of common facial pain conditions affecting $3.7-12 \%$ of the general population ${ }^{1,2}$, and is 5 times more prevalent in women 2. TMDs can be from the joint itself, the muscles of mastication, or a combination of both 3. Although up to two-thirds of these patients will seek treatment for TMDs, about $15 \%$ of them will develop into chronic disease ${ }^{1}$. According to the National Institute of Dental and Craniofacial research, the estimated annual cost incurred in the United States related to TMDs is about $\$ 4$ billion ${ }^{1}$. Because of their high prevalence, tendency to develop into chronicity, and significant financial burden to the society, TMDs are no doubt a public health problem.

The conventional approach to the management of most types of TMDs begins with conservative treatment, such as non-steroidal anti-inflammatory drugs (NSAIDs), physiotherapy, soft diet, and occlusal splint etc. ${ }^{4}$. Positive results are seen in between $75-90 \%$ of the patients with TMDs with conservative treatment ${ }^{5}$. However, there remains a small proportion of patients that are refractory to conservative treatment. In general, as the duration of pain symptoms increase, patient response to intervention diminishes ${ }^{6,7}$, and they become difficult to treat. This phenomenon applies to TMDs as it has been concluded that patients with chronic temporomandibular joint (TMJ) pain respond less favourably than in patients where the pain is non-chronic in nature ${ }^{8-10}$.

Temporomandibular joint arthrocentesis was introduced as a minimally invasive treatment for severe, limited mouth opening in the early $1990 \mathrm{~s}^{11}$. It involves lavage of the superior joint space using 2 needles with an irrigation fluid such as normal saline, 
with or without additional medications injected into the joint. While arthrocentesis has been proposed to be a first-line treatment for closed-lock of the TMJ ${ }^{12}$, different authors have also noted the effectiveness in the treatment of non-locking joints, and mechanisms have been proposed, such as removal of pathological joint fluid, and elimination of the negative pressure termed "the suction cup" effect ${ }^{8,13,14}$. Being cost-effective and safe, with a success rate of over $80 \%{ }^{15}$, arthrocentesis is now a standard procedure not only for closed lock of the TMJ, but also for TMJ arthralgia of a non-locking nature when the outcomes of conservative treatment are unsatisfactory. However, how long should conservative treatment be attempted before moving on to the next step in the treatment algorithm when results are unsatisfactory remains a question to be answered. Unfortunately, some patients are shunted between conservative treatment options for an extended period of time without observable progress, while early minimally invasive treatment, such as arthrocentesis, may have the potential to provide timely symptomatic relief as well as functional improvement. This early clinical benefit may prevent the development of chronic pain and psychological deterioration in some of the patients with TMDs. As said earlier, the difficulty of treating pain symptoms increases with the duration of those pain symptoms experienced by the patients. Therefore, the importance of understanding when to performing TMJ arthrocentesis cannot be overstated. However, there is no consensus in the literature regarding the timing of arthrocentesis with regards to conservative treatment at present.

The aim of this systematic review and meta-analysis was to determine whether timing of arthrocentesis, with regards to conservative management, has any effects on the treatment outcomes in patients with temporomandibular disorders. 


\section{METHODS}

\section{Eligibility criteria}

The following PICOTS criteria were applied:

'P' (population): adult human patients with pain due to arthrogenic, with or without myogenic TMDs. A clear clinical diagnosis or specific signs and symptoms of TMDs should be mentioned.

'I' (intervention): arthrocentesis, or lysis and lavage, with or without intra-articular injections, either as initial treatment (no prior treatment for TMDs), or as treatment after failure of conservative treatment.

' $\mathrm{C}$ ' (comparisons): control or conservative treatment only including occlusal splint, or compared to arthrocentesis with intra-articular injections, or compared to arthrocentesis with different intra-articular injections, or compared to arthrocentesis on patients with different diagnoses of TMDs

' $\mathrm{O}$ ' (outcomes): the primary outcome is pain intensity, measured by visual analogue scale (VAS) or numerical rating scale (NRS). The secondary outcome is maximum mouth opening in millimetres.

'T' (time): all studies should have a follow up period of at least 2 weeks. For those studies with arthrocentesis as treatment after failure of conservative treatment, the duration of conservative treatment should be mentioned.

'S' (study design): randomized controlled trial or controlled clinical trial or prospective clinical trial.

The following studies were excluded: studies not in English, full text not available, animal or cadaver studies, studies not related to TMDs or where there is no mention of specific 
diagnoses or signs and symptoms, studies not using arthrocentesis as intervention, studies that included less than 30 patients undergoing arthrocentesis, studies not using pain and jaw function as outcomes, non-clinical studies, retrospective studies, technical notes, case reports and case series.

\section{$\underline{\text { Information sources and search strategy }}$}

This systematic review and meta-analysis was conducted according to the PRISMA statement ${ }^{16}$. In the first round, a comprehensive electronic search in Pubmed, Cochrane Library, EMBASE (Ovid) and Scopus was updated to March 19, 2020. The following search terms were used: ("Temporomandibular Joint" OR “Temporomandibular Joint Disorders" OR "Temporomandibular Joint Dysfunction Syndrome" OR "Myofascial Pain Syndromes” OR “Craniomandibular Disorders” OR TMJ OR TMD or CMD) AND (Arthrocentesis OR “Temporomandibular Joint Arthrocentesis” OR lysis OR lavage). No restrictions on publication date, language, or status of publication were imposed. The abstracts of the articles from the computer search were reviewed for eligibility. Full texts of potentially eligible studies relevant to the treatment of TMDs by arthrocentesis were obtained and included in the second round.

In the second round, manual search was performed in 3 relevant international journals: International Journal of Oral and Maxillofacial Surgery, the Journal of Oral and Maxillofacial Surgery, and the Journal of Craniomaxillofacial Surgery. In addition, reference lists of all included studies from the first round and from the manual search were searched for relevant articles relating to management of TMDs with arthrocentesis. 
Relevant articles from the first and second rounds were included for the third-round evaluation.

In the third-round evaluation, full-texts of the included studies were evaluated based on the following inclusion criteria:

1. Prospective clinical study

2. At least 30 subjects who underwent arthrocentesis

3. Has mentioned whether arthrocentesis was initial treatment or after failure of conservative treatment

4. Has mentioned how long conservative treatment was attempted before arthrocentesis

5. Pain measured as visual analogue scale (VAS) or numerical rating scale (NRS) and maximal mouth opening in millimetres (MMO) were reported as outcome measures.

6. Has mentioned specific diagnosis or signs and symptoms

A standard form was used in the third-round evaluation of included studies. Reasons for exclusion of a study were recorded. Studies were considered eligible if all the 6 criteria mentioned above were met.

\section{$\underline{\text { Data collection process }}$}

Data from the included studies were extracted using a standard data extraction sheet specifically designed for this review. Data items that were extracted and analysed included: sample size, age at treatment, diagnosis of TMD, change in mouth opening in millimetres, change in pain measurement, duration of conservative treatment prior to 
arthrocentesis, type of conservative treatment, and adjunctive procedure with arthrocentesis, such as intra-articular injections.

\section{$\underline{\text { Risk of bias in individual studies }}$}

Two authors (D.T.S.L. and N.S.M.W.) critically appraised the studies selected from the third round for risk of bias on the following 5 aspects, which were modified from a combination of the Cochrane tool and MOOSE statement ${ }^{17}$.

1. Random allocation of subjects

2. Definition of inclusion and exclusion criteria

3. Report of losses of follow-up

4. Validated measurements

5. Statistical analysis

A study that included all these criteria was classified as having a low risk of bias.

A study that did not include one of these criteria was classified as having a moderate risk of bias

When two or more criteria were missing, the study was considered to have a high risk of bias.

When there was any discrepancy during the appraisal process between the 2 reviewers, consensus was reached with discussion.

\section{$\underline{\text { Summary measures }}$}

The variables in questions, MMO (in millimetres) and pain (VAS from 0-10), are both continuous data and the weighted mean difference (WMD) were used for analysis. When multiple values were given due to various follow-up time points, the final values were 
used for meta-analysis. Studies that used a VAS scale of 0-100 were standardized to a scale of 0-10. In studies where the range and median were given, the mean and standard deviation (SD) were estimated with statistical formulas 5 and 16 in the article by Hozo et. al. ${ }^{18}$. When the standard deviation values of only the pre-operative MMO and VAS were available, the post-operative values were assumed to be the same as the pre-operative values. When the standard deviation of both the pre-operative and post-operative values are not available, these values are excluded from the meta-analysis.

\section{Synthesis of results}

Meta-analyses were performed with the extracted WMDs of the continuous data (MMO and VAS). If a small number of studies were included, fixed effects model with $95 \% \mathrm{CI}$ was employed to construct the forest plots, otherwise, random effects model with $96 \% \mathrm{CI}$ was used. If studies of high risk of bias were found, sensitivity analysis was done after excluding those studies.

In order to access whether the timing of arthrocentesis had any effects on the clinical outcomes, we divided the included studies into 3 groups based on the timing of arthrocentesis:

1. Arthrocentesis as the initial treatment for TMDs

2. Arthrocentesis within 3 months of failing conservative treatment, and

3. Arthrocentesis after at least 3 months of conservative treatment.

The MMO and VAS of the 3 groups were compared.

All statistics were conducted using STATA (StataCorp. 2019. Stata Statistical Software: Release 16.0. College Station, TX, USA). 


\section{$\underline{\text { Risk of bias across studies }}$}

Publication bias using funnel plot were performed, as appropriate given the known limitations of these methods if the number of studies is ten or larger recommended by Cochrane handbook ${ }^{19}$.

\section{Ethical approval}

Ethical approval was not necessary as this study was a systematic review. 


\section{RESULTS}

\section{$\underline{\text { Study selection }}$}

The study selection process is shown in Figure 1. From the electronic database search, a total of 1999 articles were retrieved. After removal of duplicates, 896 articles remained. Abstract screening of the resulted articles was done, and 714 articles were excluded. A total of 182 relevant studies were included in the second-round search.

In the second-round search, manual search from 2010-2020 and reference list search from the included studies did not result in any additional articles. 182 articles were included in the third-round evaluation, where 171 articles were excluded due to failing of one or more of the inclusion criteria mentioned above. Thus, 11 studies were included in the meta-analysis.

\section{$\underline{\text { Study characteristics }}$}

The characteristics of the 11 included studies are shown in Table 1. Five RCTs, and 6 prospective clinical studies were included in the critical appraisal and meta-analysis. A total of 442 patients underwent arthrocentesis for TMDs in the 11 included studies.

The diagnoses of TMDs in the included studies included disc displacement with or without reduction (DDWR/DDWOR) (anterior or unspecified), Wilkes stages 3 and 4, internal derangement, and arthralgia.

In 4 of the included studies ${ }^{20-23}$, arthrocentesis was used as the initial treatment for TMDs. In 2 of the studies ${ }^{24,25}$, arthrocentesis was performed within 3 months of failing conservative treatment ( 2 weeks and 1 month). In the other 5 studies $^{26-30}$, arthrocentesis was performed after at least 3 months of failing conservative treatment (at least 3 months to 3 years). The duration of conservative treatment attempted ranged from 2 weeks to 3 
years in all of the studies. The types of conservative treatment included splint, soft diet, physiotherapy, and medications such as non-steroidal anti-inflammatory drugs (NSAIDs) and muscles relaxants.

Arthrocentesis was either performed without adjunctive procedures, or with intraarticular injections including hyaluronic acid (HA), platelet-rich plasma (PRP), methypredisolone acetate, tenoxicam, buprenophrine, or saline. The follow-up period in the included studies ranged from 2 weeks to 1 year.

\section{$\underline{\text { Risk of bias within studies }}$}

Assessment of the quality of the studies was done. Two studies ${ }^{22,24}$ showed a low risk of bias, 5 studies 20,21, 26, 27, 29 showed a moderate risk of bias, and 4 studies $23,25,28,30$ showed a high risk of bias. (Table 2)

\section{Synthesis of results}

We used the WMD and SD of the continuous variables for meta-analyses. In one of the studies where the range and median were given ${ }^{20}$, the mean and standard deviation were estimated with statistical formulas ${ }^{18}$. In another study, the standard deviation for the preoperative and post-operative pain measurements (VAS) were not available ${ }^{27}$, therefore, pain measurements from that study were excluded from the meta-analysis. In another study ${ }^{22}$, the VAS scores were extracted from graphs by measurement, and that the standard deviation values of the post-operative MMO and VAS were not available. The standard deviation of the post-operative MMO and VAS were assumed to be the same as the pre-operative values. 
In 3 of the studies, arthrocentesis was performed as the initial treatment for TMDs ${ }^{20-23}$, in

2 of the studies, arthrocentesis was done within 3 months of failing conservative treatment ${ }^{24,25}$, and in 5 of the studies, arthrocentesis was performed after at least 3 months of conservative treatment ${ }^{26-30}$. Both random effects model and fixed effects models were used for construction of forest plots, due to the intention of generalization inference and the high heterogeneity found in the included studies, but also because of the small number of studies included in the meta-analysis.

\section{Maximum mouth opening (MMO)}

All included studies demonstrated an improvement of MMO after arthrocentesis (ranged $3.43 \mathrm{~mm}-21 \mathrm{~mm}$ ), with the greatest improvement of MMO in the groups where arthrocentesis was performed after conservative treatment was attempted. When using fixed effects model, arthrocentesis done after 3 months of conservative treatment was found to produce the greatest improvement of MMO (WMD 13.96mm, 95\% CI: 13.18 to 14.75, $\mathrm{p}<0.001$ ), followed by arthrocentesis within 3 months (WMD $13.38 \mathrm{~mm}, 95 \% \mathrm{CI}$ : 12.73 to $13.98, \mathrm{p}<0.001$ ) (Figure 2 ). When using random effects model, similar results were obtained, with arthrocentesis done after 3 months of conservative treatment found to produce the greatest improvement of MMO (WMD $13.83 \mathrm{~mm}, 95 \% \mathrm{CI}: 10.29$ to 17.37 , $\mathrm{p}<0.001$ ), followed by arthrocentesis within 3 months (WMD $13.40 \mathrm{~mm}, 95 \%$ CI: 10.85 to $15.95, \mathrm{p}<0.001$ ) (Figure 3).

Sensitivity analysis was conducted after excluding those studies with high risk of bias ${ }^{23}$, 25, 28, 30 and showed different results. Notably, only 1 study $^{24}$ was included in the group where arthrocentesis was performed within 3 months of conservative treatment. When using fixed effects model, arthrocentesis done within 3 months of conservative treatment 
was found to produce the greatest improvement of MMO (WMD $14.70 \mathrm{~mm}, 95 \% \mathrm{CI}$ : 13.80 to $15.60, \mathrm{p}<0.001$ ), followed by arthrocentesis after 3 months (WMD $12.55 \mathrm{~mm}$, 95\% CI: 11.51 to $13.59, \mathrm{p}<0.001$ ) (Figure 4). When using random effects model in the sensitivity analysis, similar results were obtained, where arthrocentesis done within 3 months of conservative treatment was found to produce the greatest improvement of MMO (WMD $14.70 \mathrm{~mm}, 95 \%$ CI: 13.80 to 15.60 , $\mathrm{p}<0.001$ ), followed by arthrocentesis after 3 months of conservative treatment (WMD 10.17mm, 95\% CI: 7.24 to 13.11 , $\mathrm{p}<0.001$ ) (Figure 5).

\section{$\underline{\text { Pain (VAS) }}$}

All the included studies showed a reduction in pain in VAS after arthrocentesis (ranged 1.23-6.29), with arthrocentesis done within 3 months of conservative treatment producing the greatest reduction in pain, followed by arthrocentesis done after 3 months of conservative treatment. When using fixed effects model, arthrocentesis done within 3 months of conservative treatment was found to produce the greatest improvement of pain score (WMD 5.86mm, 95\% CI: 5.49 to 6.23, p<0.001), followed by arthrocentesis after 3 months (WMD 5.65mm, 95\% CI: 5.48 to 5.83, p<0.001) (Figure 6). Similar results were obtained using random effects model, with arthrocentesis done within 3 months of conservative treatment found to produce the greatest improvement of MMO (WMD $5.39 \mathrm{~mm}, 95 \%$ CI: 3.80 to $6.97, \mathrm{p}<0.001$ ), followed by arthrocentesis after 3 months (WMD 4.72mm, 95\% CI: 3.44 to 5.99, p<0.001) (Figure 7).

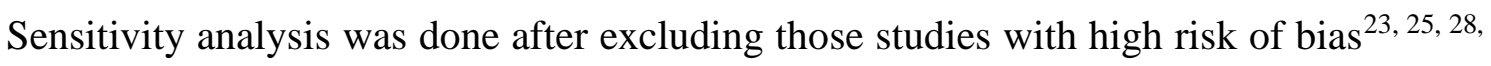
${ }^{30}$ and results were similar. When using fixed effects model, arthrocentesis done within 3 months of conservative treatment was found to produce the greatest improvement of pain 
score (WMD 6.15mm, 95\% CI: 5.74 to $6.56, \mathrm{p}<0.001$ ), followed by arthrocentesis after 3 months (WMD 3.84mm, 95\% CI: 3.34 to 4.33 , p<0.001) (Figure 8). Similar results were obtained using random effects model, with arthrocentesis done within 3 months of conservative treatment found to produce the greatest improvement of MMO (WMD $6.15 \mathrm{~mm}, 95 \%$ CI: 5.74 to $6.56, \mathrm{p}<0.001$ ), followed by arthrocentesis after 3 months (WMD 3.90mm, 95\% CI: 2.69 to 5.11, p<0.001) (Figure 9).

\section{Assessment of publication bias}

Publication bias was not assessed as there were inadequate numbers of included trials within each group to properly assess publication bias. 


\section{DISCUSSION}

At present, there is no consensus in the literature regarding the timing of arthrocentesis in the management of TMDs of arthrogenous origin. Clinical recommendations are therefore difficult to make due to this gap in the current knowledge. The results of this systematic review and meta-analysis suggested that early arthrocentesis, performed within 3 months of unsuccessful initial conservative treatment, may result in the greatest clinical benefits in terms of MMO and pain relieve. In addition, this study also suggested that a less favourable outcome may be produced when arthrocentesis is used as an initial treatment without first attempting conservative options. However, the evidence is of low to moderate quality level, as only a small number of studies were included, especially in the group where early arthrocentesis was performed. Generalizations must therefore be made with caution.

Traditional approach to management of arthrogenous TMDs usually begin with conservative treatment options, such as splint, medications, physiotherapy, and soft diet 31, 32. Surgical treatment is not offered until these conservative options have been exhausted without satisfactory improvement of pain symptoms and jaw function. This is due to the fact that many TMD symptoms do improve without any surgical treatment ${ }^{4,33}$, ${ }^{34}$. However, about 5-10\% of patients with TMDs remain unresponsive to conservative options and require surgical treatment ${ }^{35-38}$. Of all the surgical options available for TMDs, arthrocentesis is the most minimally invasive which does not cause any irreversible damage to tissues. Due to the fact that arthrocentesis has been shown to produce a more rapid clinical response than conservative treatment ${ }^{22}$, it has led to recent speculation that 
perhaps early arthrocentesis should be considered when initial conservative treatment do not show any obvious benefits.

A recent network meta-analysis of randomized clinical trials by Al-Moraissi et al. supported a paradigm shift in the management of TMDs: whereas traditional approaches embrace the use of conservative options over surgical treatment, new evidence is in favour of using minimally invasive options as initial or early treatment modalities when clear signs of improvement is not seen with conservative treatment ${ }^{39}$. These minimally invasive treatment options, especially arthrocentesis or arthroscopy with different intraarticular injections such as hyaluronic acid, platelet-rich plasma, or corticosteroids, showed a clear superiority over conservative approaches in the management of arthrogenous TMDs in terms of improving mouth opening and reducing pain ${ }^{39}$.

The introduction of arthroscopic surgery for the management of TMDs in the 1970s in japan ${ }^{40,41}$ and in the U.S. ${ }^{42,43}$ has largely replaced open-joint surgeries that were popular in the past. Although several surgical manipulations have been devised under arthroscopic view, it was realized that simple lysis and lavage also produced clinical improvement ${ }^{44}$. It was then a question whether lysis and lavage could be done blindly to simplify the procedure. The first successful report of arthrocentesis was reported by Nitzan et. al. for the treatment of closed-lock of the TMJ ${ }^{11}$. Since then, arthrocentesis has been adopt for treatment of other diagnoses of TMDs, such as painful clicking ${ }^{45}$, osteoarthritis ${ }^{46,47}$, and rheumatoid arthritis ${ }^{48}$. Although it seems that arthroscopy may be more effective in improving joint movement and reducing pain ${ }^{39,49}$, arthrocentesis has 
the advantage of being simpler, more minimally invasive, and can be done at an outpatient setting.

There were a number of limitations to this study. As mentioned earlier, there was only a limited number of studies included in this systematic review. Specifically, after exclusion of studies with high risk of bias in the sensitivity analysis, only 1 study was included in the group where arthrocentesis was performed within 3 months of failing conservative treatment. Therefore, generalization of the results of this study cannot be made with high certainty. In addition, different diagnoses of TMDs were used across studies, and it is possible that the outcomes are affected due to the inherent differences in the nature of the various diagnoses. Another shortcoming of this study was that confounding variables were present, as different intra-articular injections were used along with arthrocentesis in some of the studies, and that different modalities of conservative treatment were used. Additionally, some studies used different techniques of arthrocentesis, such as single and double-puncture arthrocentesis. Therefore, more RCTs are needed to decipher the optimal time to perform arthrocentesis in the treatment of various TMDs.

In conclusion, this study suggested that when conservative treatment fails to produce satisfactory results, early arthrocentesis may result in the greatest improvement of mouth opening and pain relieve. However, when arthrocentesis is used as an initial treatment without first attempting conservative treatment, the outcome may be less optimal than attempting conservative treatment first. Nevertheless, arthrocentesis performed at all time points produced improvement in mouth opening and pain reduction. It is important to note that the current meta-analysis only provides preliminary evidence of low to 
moderate quality level, as there is a paucity of well-designed studies in the literature regarding this topic. Therefore, more well-designed RCTs are required before we can draw more definitive conclusions. 


\section{ACKNOWLEDGEMENT}

We would like to thank Ms. Rita Suen for her great help in the screening and manual search for relevant articles.

\section{CONFLICT OF INTEREST}

None.

\section{FUNDING}

None. 


\section{REFERENCES}

1. Facial Pain. National Institute of Dental and Craniofacial Research.

2. Magnusson T, Egermark I, Carlsson GE. A longitudinal epidemiologic study of signs and symptoms of temporomandibular disorders from 15 to 35 years of age. Journal of orofacial pain 2000;14:310-319.

3. Magnusson T, Syren M. Therapeutic jaw exercises and interocclusal appliance therapy. A comparison between two common treatments of temporomandibular disorders. Swed Dent J 1999;23:27-37.

4. Rajapakse S, Ahmed N, Sidebottom AJ. Current thinking about the management of dysfunction of the temporomandibular joint: a review. Br J Oral Maxillofac Surg 2017;55:351356.

5. de Resende C, de Oliveira Medeiros FGL, de Figueiredo Rego CR, Bispo ASL, Barbosa GAS, de Almeida EO. Short-term effectiveness of conservative therapies in pain, quality of life, and sleep in patients with temporomandibular disorders: A randomized clinical trial. Cranio 2019:1-9.

6. H. M. Introduction. In: Giamberardino MA JT (ed). Pain comorbidities: understanding and treating the complex patient. Seattle: IASP Press, 2012:1-20.

7. Turk DC GR. Psychological approaches to pain management: A practitioner's hand book. New York, NY: The Gilford Press, 2002.

8. Emshoff R, Rudisch A. Determining predictor variables for treatment outcomes of arthrocentesis and hydraulic distention of the temporomandibular joint. J Oral Maxillofac Surg $2004 ; 62: 816-823$.

9. Auerbach SM, Laskin DM, Frantsve LM, Orr T. Depression, pain, exposure to stressful life events, and long-term outcomes in temporomandibular disorder patients. J Oral Maxillofac Surg 2001;59:628-633; discussion 634. 
10. Ismail F, Eisenburger M, Lange K, Schneller T, Schwabe L, Strempel J, Stiesch M. Identification of psychological comorbidity in TMD-patients. Cranio 2016;34:182-187.

11. Nitzan DW, Dolwick MF, Martinez GA. Temporomandibular joint arthrocentesis: a simplified treatment for severe, limited mouth opening. J Oral Maxillofac Surg 1991;49:11631167; discussion 1168-1170.

12. Alpaslan C, Kahraman S, Guner B, Cula S. Does the use of soft or hard splints affect the short-term outcome of temporomandibular joint arthrocentesis? Int J Oral Maxillofac Surg 2008;37:424-427.

13. Yura S, Totsuka Y. Relationship between effectiveness of arthrocentesis under sufficient pressure and conditions of the temporomandibular joint. J Oral Maxillofac Surg 2005;63:225-228. 14. Nitzan DW, Samson B, Better H. Long-term outcome of arthrocentesis for sudden-onset, persistent, severe closed lock of the temporomandibular joint. J Oral Maxillofac Surg 1997;55:151-157; discussion 157-158.

15. Monje-Gil F, Nitzan D, Gonzalez-Garcia R. Temporomandibular joint arthrocentesis. Review of the literature. Med Oral Patol Oral Cir Bucal 2012;17:e575-581.

16. Liberati A, Altman DG, Tetzlaff J, Mulrow C, Gotzsche PC, Ioannidis JP, Clarke M, Devereaux PJ, Kleijnen J, Moher D. The PRISMA statement for reporting systematic reviews and meta-analyses of studies that evaluate healthcare interventions: explanation and elaboration. BMJ 2009;339:b2700.

17. Stroup DF, Berlin JA, Morton SC, Olkin I, Williamson GD, Rennie D, Moher D, Becker BJ, Sipe TA, Thacker SB. Meta-analysis of observational studies in epidemiology: a proposal for reporting. Meta-analysis Of Observational Studies in Epidemiology (MOOSE) group. JAMA 2000;283:2008-2012.

18. Hozo SP, Djulbegovic B, Hozo I. Estimating the mean and variance from the median, range, and the size of a sample. BMC Med Res Methodol 2005;5:13.

19. Higgins JPT, Green S (editors). Cochrane Handbook for Systematic Reviews of Interventions Version 5.1.0 [updated March 2011]. The Cochrane Collaboration, 2011. 
20. Yilmaz O, Korkmaz YT, Tuzuner T. Comparison of treatment efficacy between hyaluronic acid and arthrocentesis plus hyaluronic acid in internal derangements of temporomandibular joint. J Craniomaxillofac Surg 2019;47:1720-1727.

21. Yapici-Yavuz G, Simsek-Kaya G, Ogul H. A comparison of the effects of Methylprednisolone Acetate, Sodium Hyaluronate and Tenoxicam in the treatment of nonreducing disc displacement of the temporomandibular joint. Med Oral Patol Oral Cir Bucal 2018;23:e351-e358.

22. Vos LM, Huddleston Slater JJ, Stegenga B. Arthrocentesis as initial treatment for temporomandibular joint arthropathy: a randomized controlled trial. J Craniomaxillofac Surg 2014;42:e134-139.

23. Sembronio S, Albiero AM, Toro C, Robiony M, Politi M. Is there a role for arthrocentesis in recapturing the displaced disc in patients with closed lock of the temporomandibular joint? Oral Surg Oral Med Oral Pathol Oral Radiol Endod 2008;105:274-280; discussion 281.

24. Tatli U, Benlidayi ME, Ekren O, Salimov F. Comparison of the effectiveness of three different treatment methods for temporomandibular joint disc displacement without reduction. Int J Oral Maxillofac Surg 2017;46:603-609.

25. Neeli AS, Umarani M, Kotrashetti SM, Baliga S. Arthrocentesis for the treatment of internal derangement of the temporomandibular joint. J Maxillofac Oral Surg 2010;9:350-354.

26. Toameh MH, Alkhouri I, Karman MA. Management of patients with disk displacement without reduction of the temporomandibular joint by arthrocentesis alone, plus hyaluronic acid or plus platelet-rich plasma. Dent Med Probl 2019;56:265-272.

27. Bayramoglu Z, Tozoglu S. Comparison of single- and double-puncture arthrocentesis for the treatment of temporomandibular joint disorders: A six-month, prospective study. Cranio 2019:1-6.

28. Attia HS, Mosleh MI, Jan AM, Shawky MM, Jadu FM. Age, gender and parafunctional habits as prognostic factors for temporomandibular joint arthrocentesis. Cranio 2018;36:121-127. 
29. Senturk MF, Tuzuner-Oncul AM, Cambazoglu M. Prospective short term comparison of outcomes after single or double puncture arthrocentesis of the temporomandibular joint. Br J Oral Maxillofac Surg 2016;54:26-29.

30. Prager TM, Mischkowski RA, Zoller JE. Effect of intra-articular administration of buprenorphine after arthrocentesis of the temporomandibular joint: a pilot study. Quintessence international (Berlin, Germany : 1985) 2007;38:e484-489.

31. Dimitroulis G. Temporomandibular disorders: a clinical update. BMJ 1998;317:190-194.

32. Lyons MF. Current practice in the management of temporomandibular disorders. Dent Update 2008;35:314-316, 318.

33. Lomas J, Gurgenci T, Jackson C, Campbell D. Temporomandibular dysfunction. Aust J Gen Pract 2018;47:212-215.

34. Hosgor H, Bas B, Celenk C. A comparison of the outcomes of four minimally invasive treatment methods for anterior disc displacement of the temporomandibular joint. Int $\mathrm{J}$ Oral Maxillofac Surg 2017;46:1403-1410.

35. Widmark G, Kahnberg KE, Haraldson T, Lindstrom J. Evaluation of TMJ surgery in cases not responding to conservative treatment. Cranio 1995;13:44-49.

36. Dimitroulis G. The role of surgery in the management of disorders of the Temporomandibular Joint: a critical review of the literature. Part 1. Int J Oral Maxillofac Surg 2005;34:107-113.

37. Dolwick MF, Dimitroulis G. Is there a role for temporomandibular joint surgery? Br J Oral Maxillofac Surg 1994;32:307-313.

38. Reston JT, Turkelson CM. Meta-analysis of surgical treatments for temporomandibular articular disorders. J Oral Maxillofac Surg 2003;61:3-10; discussion 10-12.

39. Al-Moraissi EA, Wolford LM, Ellis E, 3rd, Neff A. The hierarchy of different treatments for arthrogenous temporomandibular disorders: A network meta-analysis of randomized clinical trials. J Craniomaxillofac Surg 2020;48:9-23. 
40. Murakami K, Ono T. Temporomandibular joint arthroscopy by inferolateral approach. Int J Oral Maxillofac Surg 1986;15:410-417.

41. Onishi M. [Arthroscopy of the temporomandibular joint (author's transl)]. Kokubyo Gakkai Zasshi 1975;42:207-213.

42. McCain JP, Sanders B, Koslin MG, Quinn JH, Peters PB, Indresano AT. Temporomandibular joint arthroscopy: a 6-year multicenter retrospective study of 4,831 joints. J Oral Maxillofac Surg 1992;50:926-930.

43. Sanders B. Arthroscopic surgery of the temporomandibular joint: treatment of internal derangement with persistent closed lock. Oral surgery, oral medicine, and oral pathology $1986 ; 62: 361-372$.

44. Nitzan DW, Dolwick MF, Heft MW. Arthroscopic lavage and lysis of the temporomandibular joint: a change in perspective. J Oral Maxillofac Surg 1990;48:798-801; discussion 802 .

45. Yoda T, Imai H, Shinjyo Y, Sakamoto I, Abe M, Enomoto S. Effect of arthrocentesis on TMJ disturbance of mouth closure with loud clicking: a preliminary study. Cranio 2002;20:1822.

46. Nitzan DW, Price A. The use of arthrocentesis for the treatment of osteoarthritic temporomandibular joints. J Oral Maxillofac Surg 2001;59:1154-1159; discussion 1160.

47. Nitzan DW, Svidovsky J, Zini A, Zadik Y. Effect of Arthrocentesis on Symptomatic Osteoarthritis of the Temporomandibular Joint and Analysis of the Effect of Preoperative Clinical and Radiologic Features. J Oral Maxillofac Surg 2017;75:260-267.

48. Trieger N, Hoffman CH, Rodriguez E. The effect of arthrocentesis of the temporomandibular joint in patients with rheumatoid arthritis. J Oral Maxillofac Surg 1999;57:537-540; discussion 540-531.

49. Al-Moraissi EA. Arthroscopy versus arthrocentesis in the management of internal derangement of the temporomandibular joint: a systematic review and meta-analysis. Int $\mathbf{J}$ Oral Maxillofac Surg 2015;44:104-112. 


\section{Figure Captions:}

Figure 1. Study selection process.

Figure 2. Forest plot using fixed effects models showing the WMD in MMO between the 3 groups: all studies included. (Heterogeneity between studies quantified using I-squared and $p$ value of the Chi-squared test were listed.)

Figure 3. Forest plot using random effects models showing the WMD in MMO between the 3 groups: all studies included. (Heterogeneity between studies quantified using I-squared and $p$ value of the Chi-squared test were listed.)

Figure 4. Forest plot using fixed effects models showing the WMD in MMO between the 3 groups: studies with high risk of bias excluded. (Heterogeneity between studies quantified using I-squared and $\mathrm{p}$ value of the Chi-squared test were listed.)

Figure 5. Forest plot using random effects models showing the WMD in MMO between the 3 groups: studies with high risk of bias excluded. (Heterogeneity between studies quantified using I-squared and $\mathrm{p}$ value of the Chi-squared test were listed.)

Figure 6. Forest plot using fixed effects models showing the WMD in pain score in VAS between the 3 groups: all studies included. (Heterogeneity between studies quantified using Isquared and $\mathrm{p}$ value of the Chi-squared test were listed.)

Figure 7. Forest plot using random effects models showing the WMD in pain score in VAS between the 3 groups: all studies included. (Heterogeneity between studies quantified using Isquared and $\mathrm{p}$ value of the Chi-squared test were listed.) 
Figure 8. Forest plot using fixed effects models showing the WMD in pain score in VAS between the 3 groups: studies with high risk of bias excluded. (Heterogeneity between studies quantified using I-squared and $p$ value of the Chi-squared test were listed.)

Figure 9. Forest plot using random effects models showing the WMD in pain score in VAS between the 3 groups: studies with high risk of bias excluded. (Heterogeneity between studies quantified using I-squared and $p$ value of the Chi-squared test were listed.) 